\title{
Ontogenes and the Paradox of Homologous Pairing
}

\author{
B. F. Chadov, N. B. Fedorova \\ Institute of Cytology and Genetics, Siberian Branch, Russian Academy of Sciences, Novosibirsk, Russia \\ Email: boris_chadov@mail.ru
}

How to cite this paper: Chadov, B.F. and Fedorova, N.B. (2021) Ontogenes and the Paradox of Homologous Pairing. Advances in Bioscience and Biotechnology, 12, 1-9. https://doi.org/10.4236/abb.2021.121001

Received: December 4, 2020

Accepted: January 19, 2021

Published: January 22, 2021

Copyright $\odot 2021$ by author(s) and Scientific Research Publishing Inc. This work is licensed under the Creative Commons Attribution International License (CC BY 4.0).

http://creativecommons.org/licenses/by/4.0/

(c) (i) Open Access

\begin{abstract}
The mutations in ontogenes have been shown to drastically increase the nondisjunction of $\mathrm{X}$ chromosomes in the D. melanogaster meiosis. This means that ontogenes are involved in the process that brings the homologs together although both the genes and ontogenes are finally paired. The phenomenon named the paradox of homologous pairing is described. Chromosomal rearrangements (inversions and translocations) lead to formation of specific topological figures (loops and crosses) during pairing. The mutual arrangement of the nucleotide sequences of homologous ontogenes before and after formation of such figures is different. Their arrangement coincides after a figure is formed and the pairing looks homologous. However, before the figure is formed, their arrangement does not match and the pairing is actually nonhomologous. The available data on ontogenes allows this paradox to be resolved. It is assumed that the sequence of each ontogene possesses a factor that 1 ) is a product of this nucleotide sequence; 2) is co-located with this sequence; and 3) generates approaching independently of nucleotide sequence position in space. The sole candidate to the role of this factor is the DNA conformation of ontogene. The conformation in the form of a solenoid of DNA is able to generate an electromagnetic field independent of the orientation of the DNA itself. The proposed resolution of the paradox is considered in terms of the problem of genetic homology.
\end{abstract}

\section{Keywords}

Homologous Pairing, Meiosis, Ontogene, DNA Conformation, Electromagnetic Field, Drosophila

\section{Introduction}

The meiotic pairing of chromosomes is an important stage in the distribution of gene material and the very event of pairing verifies the homology of particular 
chromosomes and genes. Meiotic pairing was for a long time in the focus of attention of genetics [1] [2]; however, the nature of pairing is still vague [3]. The research into conditional mutations in drosophila [4] and the genes responsible for these mutations (ontogenes) [5] has allowed for new insight into the chromosomal pairing. The process of pairing is considered as a variant of the remote interaction of ontogenes along with a "friend-foe" recognition in the zygote [6] and interaction of ontogenes guaranteeing a bilateral symmetry [7].

Here we describe the new data on the disjunction of homologous chromosomes in drosophila meiosis. The problem of the pairing mechanism is formulated in terms of classical genetics. Characteristic of the knowledge obtained via solving problems is its high reliability and is thus of special value. The genetic problem is named "the paradox of homologous pairing". The found solution explains the mechanism of chromosome pairing and supplements the genetic theory.

According to the solution that we propose, the pairing of homologous chromosomes comprises the event of bringing together the homologous ontogenes and the event of juxtaposing the homologous sequences. Homologous ontogenes, with the same primary structure of their sequences as well as the same conformation of these sequences, are involved in driving the chromosomes together. This stage is followed by juxtaposing the nucleotide sequences of genes and ontogenes. The phenomenon of genetic homology is considered in terms of the proposed solution.

\section{Materials and Methods}

The own data of the authors on the effect of conditional mutations in the $D$. melanogaster $\mathrm{X}$ chromosome on the frequency of meiotic nondisjunction of $\mathrm{X}$ chromosomes [8] [9] [10] are used in the work along with the published data on chromosome pairing. Meiotic nondisjunction of homologous X chromosomes was studied in the females carried a conditional mutation in one of the $\mathrm{X}$ chromosomes and the In(1) Muller-5, $W^{2} B$ inversion in the other. In total, this group comprised 19 conditional mutations (Table 1). These mutations were obtained using the test for dominant lethality [4] [5] [8]. The females were mated with yellow males.

The event of meiotic nondisjunction of the $\mathrm{X}$ chromosomes in a female is indicated by emergence of patroclinous males and matroclinous females in its progeny [11]. The rate of the $\mathrm{X}$ chromosome nondisjunction is usually calculated as the ratio of doubled number of exceptional males and females to the overall progeny in a cross [2] [11]. In our case, the rate of X chromosome nondisjunction when taking into account only exceptional males was calculated as $4(\mathrm{X} 0) / \mathrm{XX}+\mathrm{XY}+4(\mathrm{X} 0)$, where $\mathrm{X} 0$ is the number of exceptional males in the progeny; $\mathrm{XX}$, number of regular females; and $\mathrm{XY}$, number of regular males. The number of exceptional males was also used to assess the share of meioses with the failure in $\mathrm{X}$ chromosome pairing. 
Table 1. Progeny of the $I n(1)$ Muller $-5, W^{2} B /+$ females carrying a conditional mutation in the $\mathrm{X}$ chromosome $(+)$ crossed with yellow males.

\begin{tabular}{|c|c|c|c|c|c|c|c|}
\hline \multirow{2}{*}{ Mutation no. } & \multicolumn{2}{|c|}{ Females } & \multicolumn{3}{|c|}{ Males } & \multirow{2}{*}{$\begin{array}{l}\text { Nondisjunction } \\
\text { of X } \\
\text { chromosomes } \\
(\%)\end{array}$} & \multirow{2}{*}{$\begin{array}{r}\text { Share of } \\
\text { abnorma } \\
\text { meioses }\end{array}$} \\
\hline & + & $B /+$ & + & $W^{2} B$ & $y$ & & \\
\hline 2 & 14 & 26 & 18 & 16 & 2 & 9.8 & 0.20 \\
\hline 3 & 19 & 23 & 14 & 14 & 4 & 21.0 & 0.37 \\
\hline 4 & 6 & 18 & 3 & 11 & 8 & 45.7 & 0.91 \\
\hline 5 & 10 & 21 & 6 & 19 & 5 & 26.3 & 0.53 \\
\hline 7 & 41 & 53 & 34 & 31 & 10 & 20.1 & 0.40 \\
\hline 8 & 20 & 25 & 18 & 17 & 4 & 16.7 & 0.33 \\
\hline 9 & 6 & 7 & 1 & 5 & 9 & 65.5 & 1.30 \\
\hline 10 & 50 & 38 & 42 & 25 & 1 & 2.5 & 0.05 \\
\hline 11 & 12 & 25 & 8 & 12 & 3 & 18.5 & 0.35 \\
\hline 29 & 39 & 37 & 19 & 38 & 15 & 31.1 & 0.62 \\
\hline 30 & 24 & 50 & 14 & 23 & 5 & 15.3 & 0.31 \\
\hline 31 & 20 & 53 & 2 & 24 & 4 & 13.9 & 0.28 \\
\hline 32 & 14 & 45 & 10 & 40 & 1 & 3.5 & 0.07 \\
\hline 33 & 27 & 40 & 25 & 34 & 9 & 22.2 & 0.44 \\
\hline 34 & 11 & 12 & 11 & 9 & 1 & 8.5 & 0.17 \\
\hline 35 & 17 & 35 & 22 & 33 & 34 & 56.0 & 1.11 \\
\hline 36 & 23 & 28 & 20 & 14 & 5 & 19.0 & 0.38 \\
\hline 38 & 24 & 25 & 30 & 29 & 5 & 15.6 & 0.31 \\
\hline 41 & 38 & 54 & 37 & 33 & 16 & 29.6 & 0.57 \\
\hline $\begin{array}{l}\text { Control (no } \\
\text { mutation) }\end{array}$ & 258 & 262 & 239 & 178 & 0 & 0 & 0 \\
\hline
\end{tabular}

The share of abnormal meioses is determined according to the number of exceptional males in the progeny as $8(\mathrm{X} 0) / \mathrm{XX}+\mathrm{XY}+4(\mathrm{X} 0)$, where $\mathrm{X} 0$ is the number of patroclinous males; $\mathrm{XX}$, number of regular females; and $\mathrm{XY}$, number of regular males. The number of patroclinous males was doubled three times in order to compensate for: 1) a 50\% loss when recording XX and X0 gametes; 2) a $50 \%$ loss when recording only $\mathrm{X} 0$ gametes as a nondisjunction (instead of $\mathrm{XX}$ and $\mathrm{X} 0$ gametes); and 3) a 50\% loss when assessing an abnormal meiosis only according to the aneuploid gametes $\mathrm{XX}$ and $\mathrm{X} 0$, since regular $\mathrm{X}$ and $\mathrm{Y}$ gametes are produced in addition to aneuploid gametes when the $\mathrm{X}$ chromosome co-orientation is lost.

\section{Results}

1) Mutations in ontogenes interfere with the pairing of homologs whereas mutations in Mendelian genes do not

The frequency of chromosome nondisjunction is an indicator of the course of 
chromosome pairing. The frequency of $\mathrm{X}$ chromosome nondisjunction in the structurally normal drosophila genotypes is $0.05 \%$ for the primary nondisjunction and $4 \%$ for the secondary nondisjunction in the XXY females [11]. As a rule, the point mutations in Mendelian genes do not change these estimates [12]. However, it appeared that the mutations in ontogenes (=conditional mutations) in the $\mathrm{X}$ chromosome drastically increase the frequency of $\mathrm{X}$ chromosome nondisjunction.

Table 1 lists the results of the experiment. In the norm, the progeny of the performed cross must comprise only regular females ( + and $\mathrm{B} /+$ ) and regular males $\left(+\right.$ and $W^{2} B$ ). The overwhelming majority of mutant females give patroclinal yellow males, suggesting formation of the $\mathrm{X} 0$ oocytes. The latter are formed as a result of nonpairing and subsequent independent segregation of the $\mathrm{X}$ chromosomes to the poles of a meiotic cell. The ability of a conditional mutation to interfere with the pairing of homologs is even more impressive when estimating the share of abnormal meioses with a loss of the $\mathrm{X}$ chromosome co-orientation (Table 1, last column).

The obtained estimates demonstrate a most pronounced disturbance of meiosis caused by the presence of a conditional mutation. A normal co-orientation of the $\mathrm{X}$ chromosomes is displaced by its absence. In several cases, the share of abnormal meiosis exceeds 1.0, which is formally impossible. Presumably, the examined females had an additional Y chromosome (XXY females), while the share was calculated using the equation intended for the primary $\mathrm{X}$ chromosome nondisjunction (XX females). The fact of emergence of XXY females in the stock is a kind of additional evidence for a high $\mathrm{X}$ chromosome nondisjunction since the XXY daughters are the result of the primary $\mathrm{X}$ chromosome nondisjunction in XX females [1] [2] [11]. The experiments with other ontogene mutations confirm the disturbed X chromosome pairing [8] [9] [10].

The current genetics distinguishes homologous and nonhomologous genetic structures (genes or chromosomes). The homologous structures have the same nucleotide composition and sequence and are able to pair, whereas the nonhomologous structures differ in the nucleotide sequence and are unable to pair. The discovered fact of the effect of the mutations in ontogenes on nondisjunction contradicts this simple and commonly recognized idea that the pairing is associated with the composition of nucleotide sequences. Involvement in the pairing appears to be dependent not only on the mere match or mismatch of two nucleotide sequences, but also on the particular genes carrying these sequences. Mutations in ontogenes interfere with the pairing unlike the mutations in Mendelian genes, which do not.

\section{2) Paradox of homologous pairing}

Figure 1 shows a pair of homologous chromosomes before pairing. The field of vision contains a segment of the chromosome; figures denote positions of nucleotides from 1 to 15. This segment is inverted in the lower homolog. Each homolog contains three genes: A (1 - 4), B (7 - 10), and C (13 - 15). Arrows show 


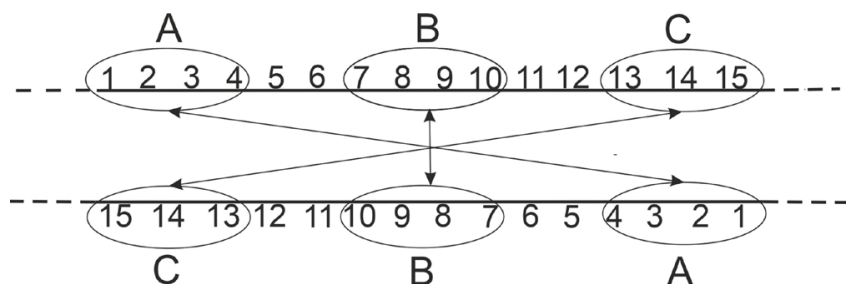

Figure 1. Juxtaposition of homologous ontogenes in a heterozygote for a chromosome inversion.

the directions of attraction of homologous genes for a bivalent to be formed.

The formation of a bivalent requires that the pairs of homologous genes (AA, $\mathrm{BB}$, and $\mathrm{CC}$ ) are formed. However, although the nucleotide sequences are the same in each pair, the formation of $\mathrm{AA}, \mathrm{BB}$, and $\mathrm{CC}$ pairs can fail because of an opposite orientation of the sequences in pairs. For example, one sequence in the AA pair is 1-2-3-4 and the other, 4-3-2-1. In terms of the current concept of homology, sequences 1-2-3-4 and 4-3-2-1 with opposite nucleotide orders are nonhomologous sequences. In this sense, the 1-2-3-4 sequence mismatches the 4-3-2-1 sequence in a similar manner as, for example, the 10-9-8-7 sequence. The paradox of the notion of homologous pairing consists in that the pairing of two identical sequences in the genomes with chromosomal rearrangements before emergence of a new topological configuration (loop, cross, and so on) is actually nonhomologous.

\section{3) Resolution of the paradox}

Despite the observed paradox, the inverted sequences nevertheless do pair. The fact of existence of unusual pairing figures suggests this particular situation. In this case, the homologous genes involved in attraction possess a "tool" or a factor that zeros out the inconvenience associated with the different positions of homologous genes at the start of pairing and guarantees their attraction independently of their mutual arrangement in space. The experimental situation makes it possible to precisely characterize this factor. The factor must 1) be a product of this nucleotide sequence (primary sequence of ontogene); 2) is co-located with this sequence (with an ontogene); and 3) be able to generate approaching independently of the position in space. Current cytogenetics suggests the sole candidate for the role of this factor-the DNA conformation in the region of the gene (conformation of ontogene). Thus, the genes of a specific category - the ontogenes - are responsible for the initiation of pairing and their specificity is determined not only by the nucleotide sequence, but also by the conformation of this sequence.

The key feature in this triad of the ontogene's properties is the generation of a remote control independently of the location in space. Ontogenes also display this ability in a "friend-foe" recognition in the zygote [6] and in the case of interaction between ontogenes that provides a bilateral symmetry. As for the "friend-foe" recognition, it is unclear whether the involved ontogenes approach each other and whether this is necessary; moreover, this is unfeasible at all in the 
case of the interaction that provides the symmetry. However, there is no doubt in all these three cases, pairing included, that the ontogenes interact in a remote manner. A physical field, specifically, an electromagnetic field, can be the basis of this interaction [7].

The hypothesis on the physical mechanism providing the approach of ontogenes implies that: 1) the DNA of ontogene is condensed forming a coil (solenoid); 2) each ontogene is a source of electromagnetic field of a certain frequency since the electric current passing through a solenoid induces electromagnetic field; and 3) the interaction of two fields of homologous ontogenes can provide their electrostatic attraction or create a field that correspondingly orient the contractile proteins involved in the approach similar to the division spindle in mitosis and meiosis.

Here, the idea of a field (generation of a remote control independently of the position in space) is coupled with the independent conclusion on the DNA conformation, which makes both assumptions more credible. The solenoids formed by DNA coils can be generators of the field. In each particular case (pairing, recognition, or provision of symmetry), electromagnetic field can induce different physical and chemical events dependent on and controlled by this field.

4) Significance of the solution for understanding the operation of a genetic system.

In accordance with the found solution, all genes are involved in the pairing of homologs; however, only ontogenes are drawn together. In this process, the spatial figure of a bivalent is formed under the effect of ontogenes, located throughout the length of the homologs. At the final pairing stage, the nucleotide sequences of both the genes and ontogenes are juxtaposed. A distinctive feature of the ontogene is a regulated coiling of the DNA string (DNA conformation). Thanks to coiling, an individual electromagnetic field of an ontogene is created, which is able to interact with the fields of the other ontogenes, both homologous and nonhomologous. A solenoid of DNA string as a generator of electromagnetic field extends its gene-specific action rather far and independently on the topology of a sequence.

The above defined role of ontogenes in pairing clarifies the question on what is homology at a genetic level. It is appropriate and valid to divide the genetic structures (genes and chromosomes) into homologous and nonhomologous ones; however, it is necessary to distinguish between the genes and ontogenes. In general, we have four types of genes with different set of properties each, namely, homologous and nonhomologous ontogenes and homologous and nonhomologous Mendelian genes. The differences between these types will be considered in a separate paper.

Ontogenes are the guardian and guarantor of the homology of a pair of chromosomes. Selection prohibits them to alter [13] [14]. Ontogenes provide pairing of the homologous chromosomes as well as "allow" the Mendelian genes within chromosomes to alter their sequences (to mutate) without any harm to the 
chromosome pairing and segregation in meiosis. If not for the conservative ontogenes, no gene constructs composed of nucleotides would stand mutagenesis.

The picture of developing chromosome synapsis has suggested cytogenetics that pairing comprises two phases: approach of homologs and precise pairing [3]. The obtained data on the distinct roles of genes and ontogenes in pairing confirms the properness of this division. Ontogenes provide the process of approach and both the genes and ontogenes are involved in the precise pairing. The co-orientation of nonhomologous chromosomes takes place in drosophila meiosis [2] [15]; some authors regard this co-orientation as a proof for existence of a specific distributive pairing [16] [17]. The role of ontogenes in the coorientation of nonhomologs in this case is most likely.

The idea on generation of electromagnetic field is only a hypothesis. However, this is a component of the proposed solution and thus is a strong argument. The field is necessary not only for the pairing of homologs, but also for a "friend-foe" recognition in the zygote [6] and provision of a bilateral symmetry [7]. The genes associated in their operation with the orientation in space are also necessary for the control of ontogenesis. As is shown, ontogenes "supervise the architectural styling of the cell mass of a developing organism" [7]; correspondingly, the idea of an electromagnetic field is most promising for the development of the theory of ontogenesis.

\section{Conclusions}

The discovery of a new category of genes - ontogenes has not changed the keystone of genetics on generation of the traits of a living organism with the help of discrete genes (DNA regions) but has demonstrated that the Mendelian genes alone are insufficient to construct the organism. Ontogenes are necessary for the control of Mendelian genes and formation of cell ensembles. The modus operandi of the ontogenes is also specific. Here we demonstrate by the case study of homologous chromosomes that the ontogenes in their operation use the varying folding (conformation) of the nucleotide sequence along with the sequence itself. It is assumed that the conformation is involved in the generation of an electromagnetic field, which underlies the remote control implemented by the ontogenes. The premise that electromagnetic fields exist in living matter is not new; however, in our case, it is a component of the solution of a particular genetic problem, which makes it more credible.

Numerous papers describe the chromosome pairing. The zygomere hypothesis reflects the fact of existence of individual chromosome regions that initiate pairing [3]. It is unlikely that the phenomenon that we named here "the paradox of homologous pairing" has not been noticed as well. However, any logically consistent picture of the gene interaction during pairing has not been devised. Now, it is clear that this picture cannot be constructed in principle based on the classical concept of a universal gene. The situation changes once the conditional mutations in drosophila are discovered, the concept of two-component genome 
is proposed, and a strong effect of mutations in ontogenes on chromosome disjunction is observed.

\section{Acknowledgements}

The authors thank the institute of cytology and genetics, siberian branch, russian academy of sciences, for financial support of this work (budget project no. 0259-2021-0011).

\section{Conflicts of Interest}

The authors declare no conflicts of interest regarding the publication of this paper.

\section{References}

[1] Morgan, T.H., Bridges, C.B. and Sturtevant, A.H. (1925) The Genetics of Drosophila. 's-Gravenhage Martinus Nijhoff.

[2] Chadov, B.F. (1991) From the Phenomenon of Nondisjunction to the Problem of Chromosome Co-Orientation (To the Commemoration of Bridges Paper Published 75 Years Ago). Genetika, 27, 1877-1903. (In Russian)

[3] Sybenga, J. (1966) The Zygomere as Hypothetical Unit of Chromosome Pairing Initiation. Genetica, 37, 186-198. https://doi.org/10.1007/BF01547130

[4] Chadov, B.F., Chadova, E.V., Kopyl, S.A. and Fedorova, N.B. (2000) A New Class of Mutations in Drosophila melanogaster. Doklady Biological Sciences, 373, 423-426.

[5] Chadov, B.F., Fedorova, N.B. and Chadova, E.V. (2015) Conditional Mutations in Drosophila melanogaster. On the Occasion of the 150th Anniversary of Mendel, G.'s Report in Brünn. Mutation Research/Reviews in Mutation Research, 765, 40-55. http://dx.doi.org/10.1016/j.mrrev.2015.06.001

[6] Chadov, B.F., Chadova, E.V. and Fedorova, N.B. (2017) A Novel Type of Gene Interaction in D. melanogaster. Mutation Research/Fundamental and Molecular Mechanisms of Mutagenesis, 795, 27-30. https://doi.org/10.1016/j.mrfmmm.2017.01.002

[7] Chadov, B.F. and Fedorova, N.B. (2019) The Mutations Disturbing the Bilateral Symmetry in Drosophila. SCIOL Genetic Science, 2, 139-152.

[8] Chadov, B.F. (2001) Mutations Capable of Inducing Speciation. In: Stegnij, V.N., Ed., Evolution Biology, Tomsk State University Press, Tomsk, 138-162. (In Russian) http://www.evolbiol.ru/

[9] Chadov, B.F. (2002) “The Image" of the Regulatory Gene in Experiments with Drosophila. Russian Journal of Genetics, 38, 869-880.

https://doi.org/10.1023/A:1016356418121

[10] Chadov, B.F., Chadova, E.V., Khotskina, E.A., Artemova, E.V. and Fedorova, N.B. (2004) The Main Effect of Chromosomal Rearrangement Is Changing the Action of Regulatory Genes. Russian Journal of Genetics, 40, 723-731. https://doi.org/10.1023/B:RUGE.0000036520.93159.a5

[11] Bridges, C.B. (1916) Non-Disjunction as Proof of the Chromosome Theory of Heredity. Genetics, 1, 1-52.

[12] Chadov, B.F. (1971) Nonhomologous Chromosome Pairing in the Oogenesis of Drosophila melanogaster, Extended Abstract of Nonhomologous Chromosome 
Pairing in the Oogenesis of Drosophila melanogaster Dissertation. Institute of Developmental Biology, Moscow. (In Russian)

[13] Chadov, B.F. and Fedorova, N.B. (2018) Zygotic Selection in Drosophila melanogaster and a New Edition of Darwin's Concept of Speciation. Proceedings of VInternational Symposium, Tomsk, 12-16 November, 49-51. (In Russian)

[14] Chadov, B.F., Chadova, E.V. and Fedorova, N.B. (2019) Ontogenes and the Problem of Speciation. Journal of Evolutionary Science, 1, 33-47. https://doi.org/10.14302/issn.2689-4602.jes-18-2431

[15] Cooper, K.W., Zimmering, S. and Krivshenko, J. (1955) Interchromosomal Effects and Segregation. Proceedings of the National Academy of Sciences of the United States of America, 41, 911-914. https://doi.org/10.1073/pnas.41.11.911

[16] Grell, R.F. (1964) Chromosome Pairing, Crossing over and Segregation in Drosophila melanogaster. National Cancer Institute Monograph, 18, 215-242.

[17] Grell, R.F. (1976) Distributive Pairing. In: Ashburner, M. and Novitski, E., Eds., The Genetics and Biology of Drosophila, Academic Press, London, New York, San Francisco, 435-486. 